\title{
Antibacterial Activity in Pyrimidine Derivatives in Lab, Using Agar Well-diffusion Method - An Update on Novel Research
}

\author{
Sudha Singh ${ }^{1}$, Shamim Ahmad ${ }^{1}$, Devansh Mehta ${ }^{1,}$, Shamsher Alam ${ }^{2}$ \\ ${ }^{1}$ Department of Pharmaceutical Technology, Translam Institute of Pharmaceutical Education and Research, Meerut, India \\ ${ }^{2}$ Department of Pharmaceutical Chemistry, College of Pharmacy, Jazan Universiy, Jazan, Saudi Arabia \\ Email address: \\ sudha.singh758@gmail.com (S. Singh),shamim2009in@gmail.com (S. Ahmad),devanshm84@gmail.com (D. Mehta), \\ wshams.alam@gmail.com (S. Alam) \\ ${ }^{*}$ Corresponding author
}

\section{To cite this article:}

Sudha Singh, Shamim Ahmad, Devansh Mehta, Shamsher Alam. Antibacterial Activity in Pyrimidine Derivatives in Lab, Using Agar Welldiffusion Method - An Update on Novel Research. Pharmaceutical Science and Technology. Vol. 3, No. 2, 2020, pp. 40-47.

doi: $10.11648 /$ j.pst.20190302.12

Received: November 22, 2019; Accepted: January 2, 2020; Published: February 14, 2020

\begin{abstract}
Pyrimidine derivatives have been in use since ages. Pyrimidine derivatives have shown wide range of pharmacological actions. However still many activities haven't been researched so far. It is because of this reason the derivatives of the pyrimidine compounds was selected for the present study. In the present research work, it is all about the newer compounds being developed, designed, and experimented for their pharmacological actions. The current research shows the antibacterial activity tested in the new compounds designed in the laboratory. The antimicrobial activities were tested using agar plate and well diffusion method was especially selected for the present research work. Complete and proper steps were followed as per the guidelines. All precautions were taken while carrying out research for the derivative compounds in the present study. The designed compound showed effective and positive antibacterial activity present in the compound designed in the laboratory. Activity on both Gram positive and Gram negative microbes was tested, and the outcomes were in affirmation of the derivative compounds to be studied further for their pharmacological activities on both Gram positive and Gram negative microbes, and the outcomes were in affirmation of the derivative compounds to be studied further for their pharmacological activities for the derivative compounds selected in the present study. As a researcher, it is recommended to carry out further research on the same compound and being tested for other pharmacological activities.
\end{abstract}

Keywords: Antibacterial Activity, Pyrimidine, Toxicity

\section{Introduction}

Medicinal chemistry is a chemistry-based discipline involving features of biological, medical and pharmaceutical sciences. It is concerned with the invention, discovery, design, and identification of biologically active compounds. It is also concerned with the study of their metabolism, the interpretation of their mode of action at the molecular level, and the construction of their quantitative structural activity relationships (QSARs), which is the relationship between chemical structure and its pharmacological aspect [1].

Although there has been a great deal of success in understanding the relationship between the chemical structure and the biological activities in numerous areas, especially for antibacterial drugs, there are still many human afflictions that requires new and improved drugs [2].

When a new pharmaceutical lead compound is discovered, extensive and costly efforts are usually made to prepare a series of analogues so that better activity can be found. The metabolism of the drug is an important object of study in the medicinal chemistry and considerable efforts needs to be spent on detailed analysis of bioconversions, a new drug series undergoes. Modern analytical methods such as mass spectrophotometery permit the identification of minute quantities of metabolites. The intellectual goal of the medicinal chemistry is to determine the mode of action 
(MOA) of drugs at the molecular level. The objective of medicinal chemistry is the design and production of compounds that can be used in medicine for the prevention, treatment and cure of humans or animal diseases [3].

Five and six-membered heterocyclic nitrogen containing systems such as pyrazoles, imidazoles, triazoles, thiozolidines, pyrazolidines, piperidines, oxane pyrimidines, pyridines, thianes, and pyran'sas far arethe most important in the ongoing search for more efficacious drugs in the fields of antibacterials, antifungals, antituberculars, anti-inflammatory, diuretics, antirheumatics, and antihistaminics. Nitrogencontaining heterocyclic compounds have received considerable attention due to their wide range of pharmacological activity. Pyrimidine and their derivatives are considered as highly important class for medicinal drugs as well. As pyrimidine is a basic nucleus in DNA \& RNA, it has been found to be associated with diverse biological activities. Pyridine, a heterocyclic nucleus, hasplayed a pivotal role in the development of different medicinal agents. Current studies have demonstrated that pyridine congeners are associated with different biological activities, such as pesticidal, fungicidal and antibacterial activity. Pyrimidines and pyridines have contributed to the diverse library of compounds demonstrating selective affinity to the 5-HT7 receptor. Pyrimidines are six-member heterocyclic rings, containing two nitrogen atoms on the 1,3 positions, as depicted as (figure 1).

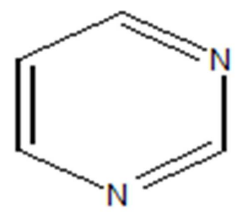

Figure 1. Pyrimidine Structure.

Pyrimidines are present among the three isomeric diazines. Several pyrimidines mainly cytosine (I), uracil (II) and thymine (III) have been isolated from the nucleic acid hydrolysis as shown in Figure 2.<smiles>Nc1cc[nH]c(=O)n1</smiles>

(I)

Figure 2. Cytosine \& Uracil Structures.

The nucleic acid are essential constituent of all cell and thus of all living matter cytosine is found to be present in both types of nucleic acids i.e. ribonucleic acid (RNA) and deoxyribonucleic acid (DNA) [4].

In addition to this, Pyrimidines ring is also found in Vitamin $\mathrm{B}_{1}$, Barbituric acid (IV) and its several derivatives e.g. Veranal (V) which are used as Hypnotics (Figure 3) [5].

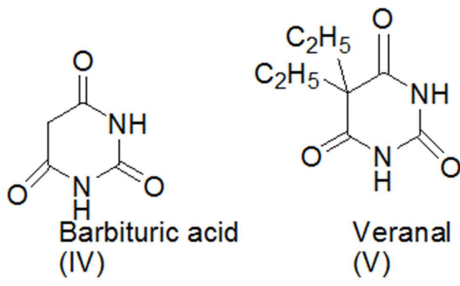

Figure 3. Barbituric Acid \& Veranal Structures.

Numerous reports have appeared in the literature, that highlight chemistry and uses of pyrimidines, and their derivatives likeSulfadiazines, Sulfamerazines, and Sulfamethazines. These agents are inhibitors of folic acid biosynthesis in microorganism. Pyridine is a ubiquitous chemical compound. The aromatic, monocyclic azine is utilized as a reagent or as a polar aprotic solvent. It is salient in a number of biological systems and industrial applications. Naturally occurring pyridines include the nicotinamides, a component of the vitamin B group. Pyridines are precursors to various pharmaceuticals, adhesives, agrichemicals, and synthetic pigments. A pyrimidine has many properties in common with pyridine, as the number of nitrogen atoms in the ring increases, the ring pi electrons become less energetic and electrophilic aromatic substitution gets more difficult while nucleophilic aromatic substitution gets easier [6].

\section{Synthesis of Pyrimidine}

Several approaches are available for synthesis of pyrimidines and are as follows:

a). Synthesis from Enamines, Triethylorthoformate

A $\mathrm{ZnCl}_{2}$-catalyzed three-component coupling reaction allows the synthesis of various 4, 5-disubstituted pyrimidine derivatives in a single step from functionalized enamines, triethylorthoformate, and ammonium acetate. The procedure can be successfully applied in efficient synthesis of monoand disubstituted pyrimidine derivatives, using methyl ketone derivatives instead of enamines (as shown in figure 4) [7]

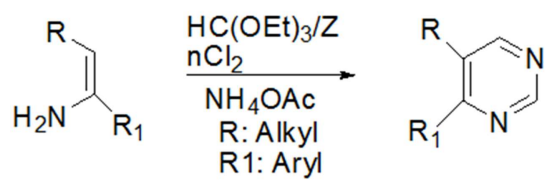

Figure 4. Coupling Reactions.

\section{b). Synthesis from $N$-vinyl/aryl Amides}

The direct condensation of cyanic acid derivatives with $\mathrm{N}$ /aryl amides affords the corresponding C4-heteroatom substituted pyrimidines (as shown in figure 5) [8].

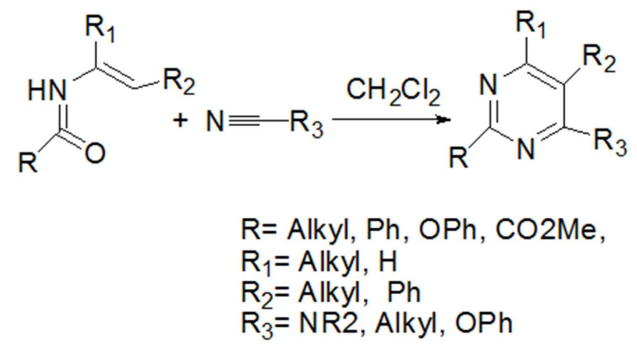

Figure 5. Condensation Reaction resulting in respective derivative. 
c). Synthesis of Pyrimidine from $\beta$-formyl Enamides

A novel and efficient synthesis of pyrimidine from $\beta$-formyl enamide involves samarium chloride, catalyzed cyclisation of $\beta$-formyl enamides, using urea as source of ammonia under microwave irradiation (as shown in Figure 6) [9].

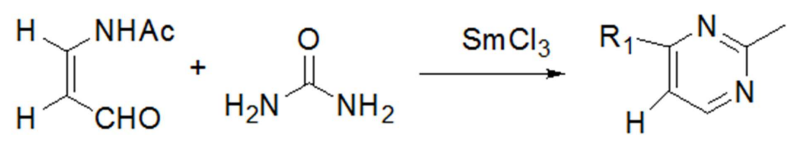

Figure 6. Synthesis Reaction.

d). Synthesis from Activation of Amide with 2Chloropyridine

A single-step conversion of various $\mathrm{N}$-vinyl and $\mathrm{N}$-aryl amides to the corresponding pyrimidine and quinazoline derivatives, involves amide activation with 2-chloropyridine and trifluoromethanesulfonic anhydride followed by nitrile addition into the reactive intermediate and cycloisomerization (as shown in figure 7 [10]).

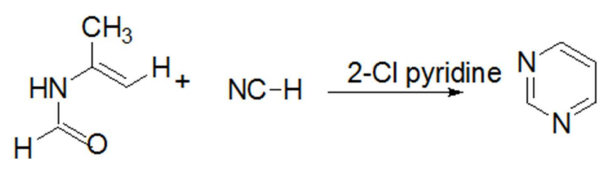

Figure 7. Cycloisomerization Reactions.

\section{Synthetic Reaction}

The materials and methods followed for the present research has been carried out as per the required guidelines, and after reviewing the various review and research papers. The pyrimidine derivatives designed in the present research were identified at molecular level using various spectrophotometric analysis.

The antimicrobial presence was tested using guidelines and instructions after reviewing various research and review papers. All specific laboratory equipment's were used to carry out the research effectively and efficiently.
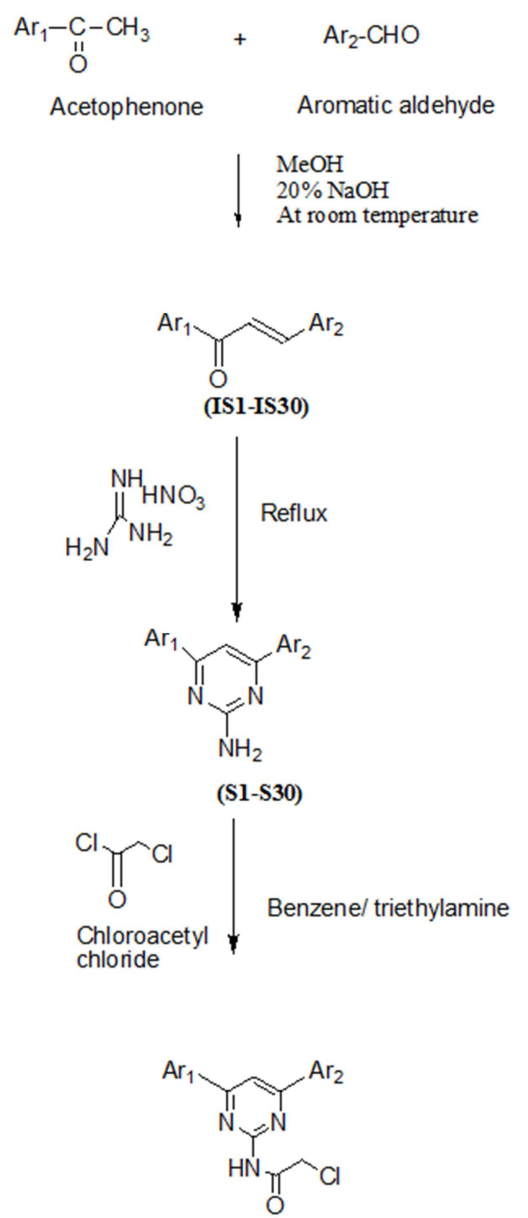

(P1-P30)

Figure 8. Scheme. 1

\section{Spectral Characterizations of Synthesized Pyrimidine Derivatives}<smiles>CC(=O)Nc1nc(-c2ccccc2)cc(-c2ccc(Cl)cc2Cl)n1</smiles>
4-(2,4-dichlorophenyl)-6phenylpyrimidin-2-amine
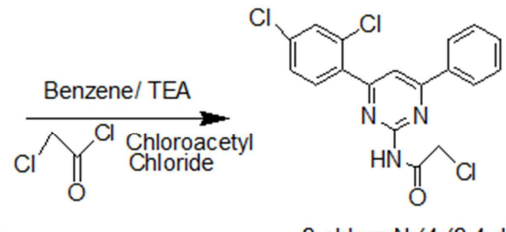

2-chloro-N-(4-(2,4-dichlorophenyl)-6phenylpyrimidin-2-yl)acetamide

Figure 9. Synthesis of 2-chloro-N-(4-(2, 4-dichlorophenyl)-6-phenylpyrimidin-2-yl) acetamide (P1).<smiles>Nc1nc(-c2ccccc2)cc(-c2cc(Cl)ccc2Cl)n1</smiles>
pyrimidin-2-amine

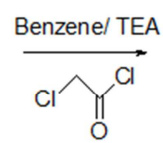

Chloracetyl Chloride

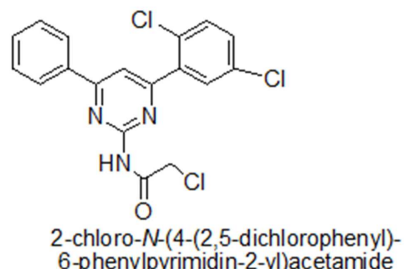

Figure 10. Synthesis of 2-chloro-N-(4(-2, 5-dichlorophenyl)-6-phenylpyrimidine-2-yl) acetamide (P2). 
$\mathrm{Br}$

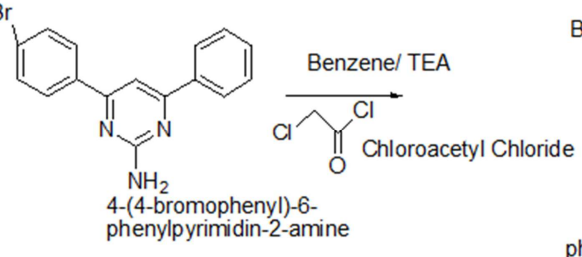

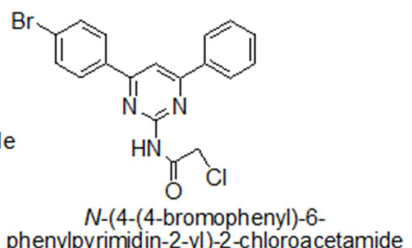

phenylpyrimidin-2-yl)-2-chloroacetamide

Figure 11. Synthesis of N-(4-(4-bromophenyl)-6-phenylpyrimidin-2-yl)-2-chloroacetamide (P3).<smiles>Nc1cccc(-c2cc(-c3ccccc3)nc(N)n2)c1</smiles><smiles>COc1ccccc1OC(=O)OC(CCl)C(=O)CCl</smiles><smiles>Nc1cccc(-c2cc(-c3ccccc3)nc(NC(=O)CCl)n2)c1</smiles>
phenylpyrimidin-2-amin

$\mathrm{N}$-(4-(3-aminophenyl)-6phenylpyrimidin-2-yl)-2chloroacetamide

Figure 12. Synthesis of $\mathrm{N}$-(4-(3-aminophenyl)-6-phenylpyimidine-2-yl)-2-chloroacetamide (P4).<smiles>Nc1cccc(-c2cc(-c3ccccc3)nc(N)n2)c1</smiles>

4-(3-aminophenyl)-6-phenyl pyrimidin-2-amine

\section{Benzene/ TEA}<smiles>ClCC(Cl)=C(Cl)CCl</smiles><smiles></smiles>

N-(4-(3-aminophenyl)-6-phenylpyrimidin-2-yl)

2-chloroacetamide

Figure 13. Synthesis of $N$-(4-(3-aminophenyl)-6-phenylpyrimidin-2-yl)-2-chloroacetamide (P5).

Table 1. Spectral Characterizations of Synthesized Pyrimidine Derivatives.

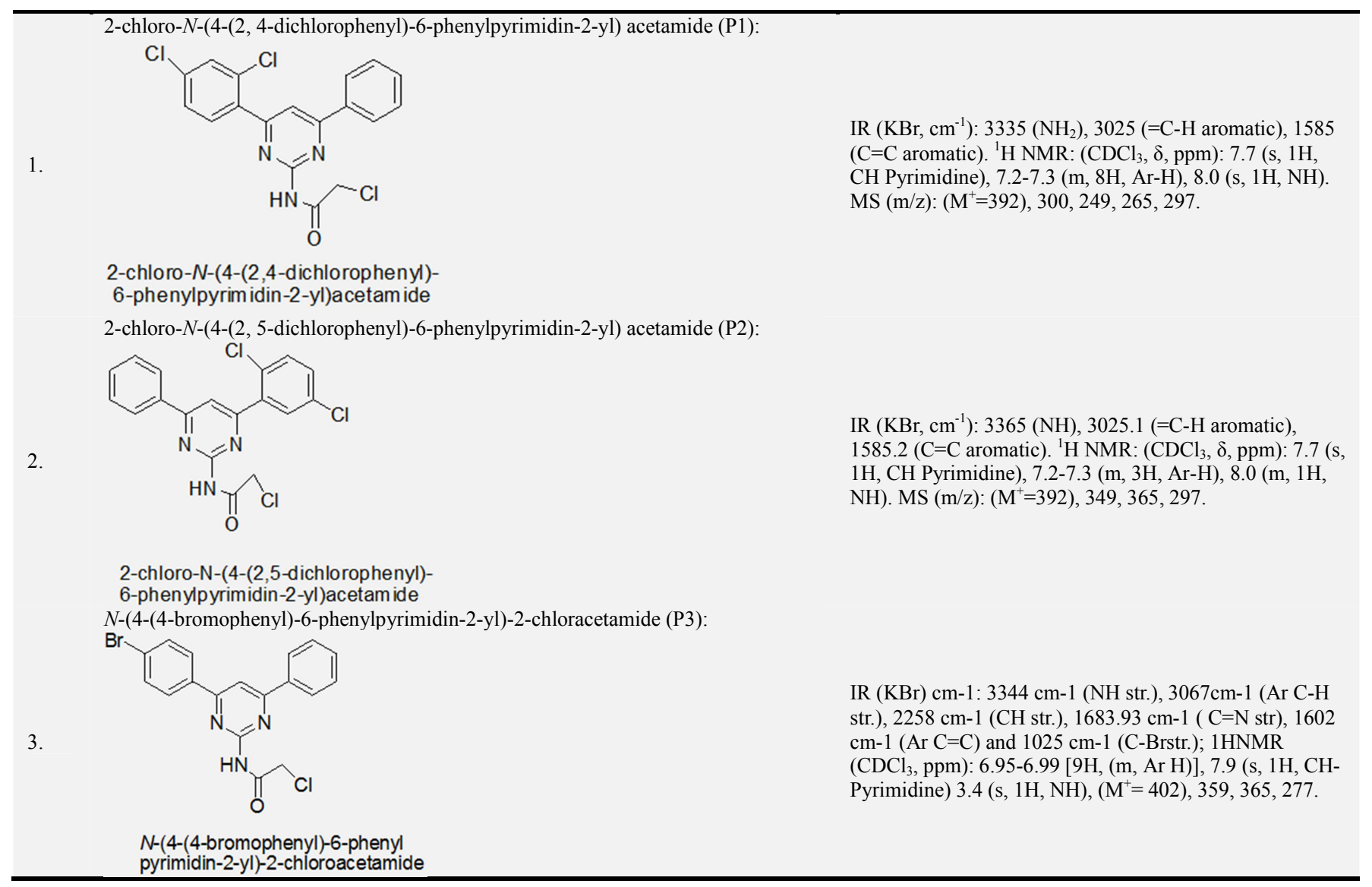




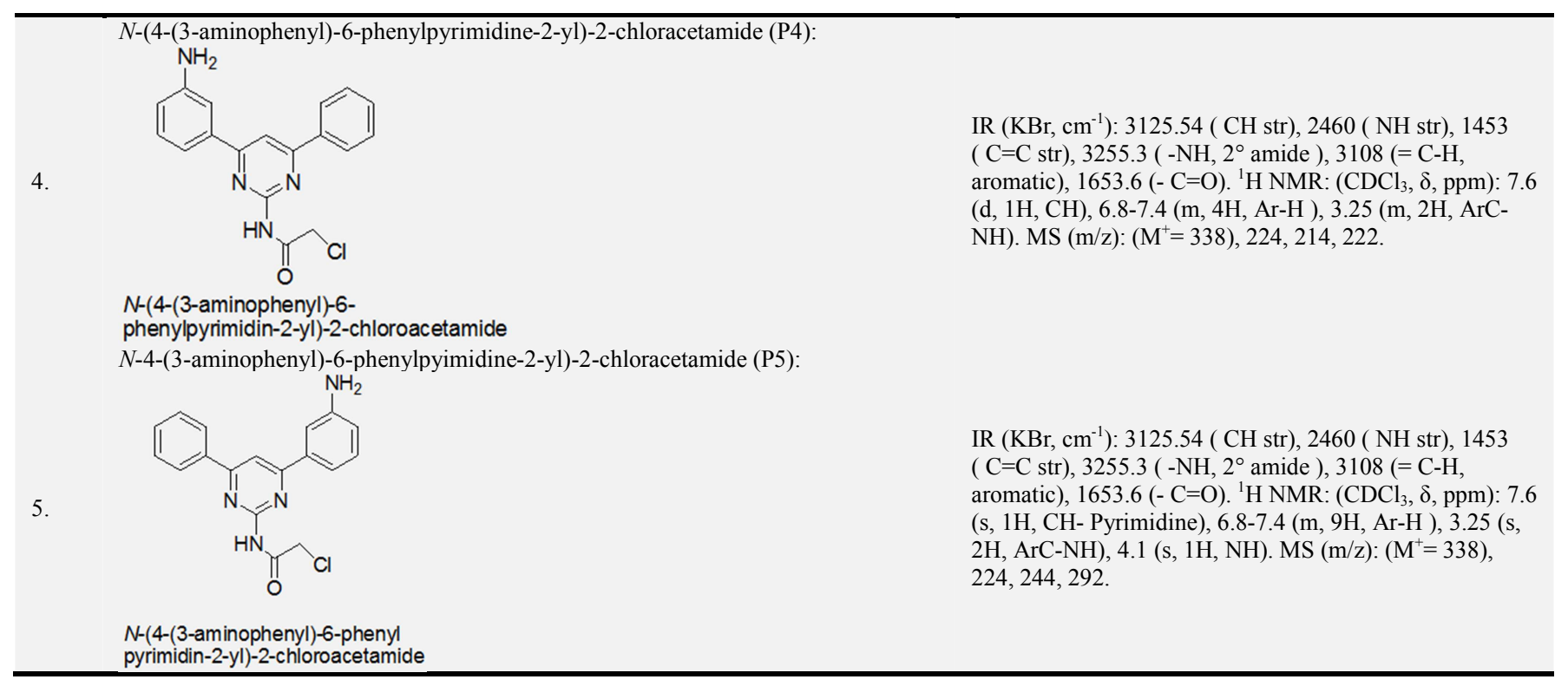

\section{Antimicrobial Activity}

Paul Ehrlich, developed the concept of chemotherapy to treat the microbial diseases. According to Paul Ehrlich, the pathogens can be destroyed under the action of chemotherapeutic agents in such a way that the host cell is not harmed. The concept of antibiotics was confirmed by Pasteur in 1877 . The development of bacterium anthrax in urine, was inhibited by the airborne bacteria. In the late 1930s the Sulphadrugs, gained much significance. The first ever antibiotic was discovered by Alexander Fleming, and the name of the antibiotic was Penicillin; the discovery of which was made in the year 1929. In the year 1940 the first clinical trial of Penicillin was completed by Domagk in 1935. [11] Protonsil is a suphonamide dye in the pyrogenic infections and soon it was realized that para amino benzene sulphonamide was the active moiety, and its essential feature was not the dye. Sulfa pyridine was the first sulfonamide. In 1940 Waksman and his colleagues, undertook a regular search of actinomycetes as a source of antibiotics, and discovered Streptomycin in 1944. This group of soil microbes, proved to be rich house of antibiotics and soon Tetracyclines, Chloramphenicol, Erythromycin and many others followed. [12] Many current Antifungal are available, the two most important antibiotics of which are; a. Amphotericin-B to deal with systemic mycosis, and b. Grseiofulvin to supplement attack on dermatophytes. They were introduced in and around the year
1960. Antifungal property of Flucytosine was famous in 1970. But it could serve only as evaluation drug to Amphotericin. The development of imidazoles in mid 1970's and triazoles 1980's has been advancement as of now. [13]

\subsection{Methodology of Antimicrobial Activity}

The well diffusion test was performed using Agar medium, as per the procedure described by Magaldi et al 2004 and the medium was autoclaved at $15 \mathrm{lbs}$ pressure $121^{\circ} \mathrm{C}$ for $15 \mathrm{~min}$ then was immediately cooled to $50-55^{\circ} \mathrm{C}$ in a water bath after removing it from the autoclave. The cooled medium was poured into sterile petridishes to a uniform depth of $4 \mathrm{~mm}$, this is equivalent to approximately $25 \mathrm{ml}$ in a $90 \mathrm{~mm}$ plate. Once the medium had solidified then the culture was inoculated on the medium. Within 15 min of adjusting the densityof the inoculums a sterile cotton swab was dipped into the standardized bacterial and yeast suspension or inoculated with $1 \mathrm{ml}$ of the organism suspension. [14]

The sterile swab was used on the surface of the Agar medium to ensure an even distribution of the inoculums. The plates were undisturbedfor 3 to 5 minutes to ensure absorption of excess moisture.

Sterilized $9 \mathrm{~mm}$ cork borer was used to make agar well, $100 \mu \mathrm{L}$ of the diluted compound stocl solution were placed into each wells and 100\% DMSO used ass control. [15]

\section{\%Inhibition= I (dia of inhibition zone in mm) / 90(dia of Petri plates in mm) $\times 100$}

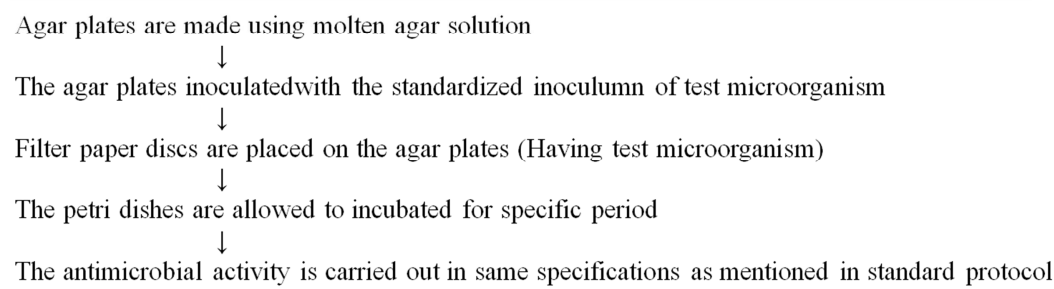

Figure 14. Methodology for the research. 


\begin{tabular}{lcl}
\hline \multicolumn{3}{l}{ Toxicity (Predicted Activity through model) } \\
\hline $\begin{array}{l}\text { Tetrahymena } \\
\text { Pyriformis Toxicity }\end{array}$ & 1.1746 & $\mathrm{pIGC}_{50}, \mathrm{ug} / \mathrm{L}$ \\
\hline
\end{tabular}

\subsection{3. $\mathrm{N}$-(4-(4-bromophenyl)-6-phenylpyrimidin-2-yl)-2- chloracetamide (P3)}<smiles>O=C(CCl)Nc1nc(-c2ccccc2)cc(-c2ccc(Br)cc2)n1</smiles>

$\mathrm{N}$-(4-(4-bromophenyl)-6-phenyl pyrimidin-2-yl)-2-chloroacetamide

Figure 18. Structure of Molecule P3.

Table 5. P3: Toxicity.

\begin{tabular}{lll}
\hline P3: Toxicity (Qualitative Prediction \& Probability) & \\
\hline Human Ether-a-go-go-Related & Weak inhibitor & 0.9449 \\
Gene Inhibition & Non-inhibitor & 0.8359 \\
AMES Toxicity & Non AMES toxic & 0.8024 \\
Carcinogens & Non-carcinogens & 0.8766 \\
Fish Toxicity & High FHMT & 0.8046 \\
Tetrahymena Pyriformis & High TPT & 0.9934 \\
Toxicity & Low HBT & 0.8942 \\
Honey Bee Toxicity & Not ready biodegradable & 1.0000 \\
Biodegradation & III & 0.6906 \\
Acute Oral Toxicity & Non-required & 0.5920 \\
\hline Carcinogenicity (Three-class) &
\end{tabular}

\begin{tabular}{lll}
\hline \multicolumn{3}{l}{ Toxicity (Predicted Activity through model) } \\
\hline Rat Acute Toxicity & 1.9770 & $\mathrm{LD}_{50}, \mathrm{~mol} / \mathrm{kg}$ \\
Fish Toxicity & 1.3860 & $\mathrm{pLC}_{50}, \mathrm{mg} / \mathrm{L}$ \\
Tetrahymena Pyriformis Toxicity & 1.2935 & $\mathrm{pIGC}_{50}, \mathrm{ug} / \mathrm{L}$ \\
\hline
\end{tabular}

\subsubsection{N-(4-(3-Aminophenyl)-6-Phenylpyrimidine-2-yl)-2- Chloracetamide $(\mathrm{P4})$}<smiles>Nc1cccc(-c2cc(-c3ccccc3)nc(NC(=O)CCl)n2)c1</smiles>

$\mathrm{N}$-(4-(3-aminophenyl)-6-

phenylpyrimidin-2-yl)-2-chloroacetamide

Figure 19. Structure of Molecule P4.

Table 6. P4: Toxicity.

\begin{tabular}{lll}
\hline P4: Toxicity (Qualitative Prediction \& Probability) & \\
\hline Human Ether-a-go-go-Related Gene & Weak inhibitor & 0.9861 \\
Inhibition & Non-inhibitor & 0.7675 \\
AMES Toxicity & Non AMES toxic & 0.6509 \\
Carcinogens & Non-carcinogens & 0.8498 \\
Fish Toxicity & High FHMT & 0.5856 \\
Tetrahymena Pyriformis Toxicity & High TPT & 0.8870 \\
Honey Bee Toxicity & Low HBT & 0.8997 \\
Biodegradation & Not ready & 1.0000 \\
\hline
\end{tabular}

\begin{tabular}{lll}
\hline \multicolumn{3}{l}{ P4: Toxicity (Qualitative Prediction \& Probability) } \\
\hline \multicolumn{3}{c}{ biodegradable } \\
Acute Oral Toxicity & III & 0.6434 \\
Carcinogenicity (Three-class) & Non-required & 0.5918 \\
\hline \multicolumn{3}{l}{} \\
\hline \multicolumn{3}{l}{ Toxicity (Predicted Activity through model) } \\
\hline Rat Acute Toxicity & 2.1034 & $\mathrm{LD}_{50}, \mathrm{~mol} / \mathrm{kg}$ \\
Fish Toxicity & 1.6855 & $\mathrm{pLC}_{50}, \mathrm{mg} / \mathrm{L}$ \\
Tetrahymena Pyriformis Toxicity & 0.5689 & $\mathrm{pIGC}_{50}, \mathrm{ug} / \mathrm{L}$ \\
\hline
\end{tabular}

\subsubsection{N-4-(3-Aminophenyl)-6-Phenylpyimidine-2-yl)-2-} Chloracetamide (P5)<smiles>Nc1cccc(-c2cc(-c3ccccc3)nc(NC(=O)CCl)n2)c1</smiles>

$N$-(4-(3-aminophenyl)-6-phenyl pyrimidin-2-yl)-2-chloroacetamide

Figure 20. Structure of Molecule P5.

Table 7. P5: Toxicity.

\begin{tabular}{lll}
\hline P5: Toxicity (Qualitative Prediction \& Probability) \\
\hline Human Ether-a-go-go-Related Gene & Weak inhibitor & 0.9864 \\
Inhibition & Non-inhibitor & 0.7266 \\
AMES Toxicity & Non AMES toxic & 0.6086 \\
Carcinogens & Non-carcinogens & 0.8529 \\
Fish Toxicity & High FHMT & 0.6570 \\
Tetrahymena Pyriformis Toxicity & High TPT & 0.8345 \\
Honey Bee Toxicity & Low HBT & 0.9253 \\
Biodegradation & Not ready & 1.0000 \\
Acute Oral Toxicity & biodegradable & 0.5720 \\
Carcinogenicity (Three-class) & NII & 0.6607 \\
\hline \multicolumn{4}{l}{} \\
\hline Toxicity (Predicted Activity through model) \\
\hline Rat Acute Toxicity & 0.1951 & $\mathrm{LD}_{50}, \mathrm{~mol} / \mathrm{kg}$ \\
Fish Toxicity & 1.5257 & $\mathrm{pLC}_{50}, \mathrm{mg} / \mathrm{L}$ \\
Tetrahymena Pyriformis Toxicity & 0.7351 & $\mathrm{pIGC}_{50}, \mathrm{ug} / \mathrm{L}$ \\
\hline
\end{tabular}

\section{Result and Discussion}

A novel series of compounds (P1-P5) were synthesized and characterized. The Infrared spectra of compounds (P1P5) revealed absorption bands within $3770-3357 \mathrm{~cm}^{-1}$ for (NH str), 1608- $1585 \mathrm{~cm}^{-1}$ for $\mathrm{C}=\mathrm{N}$. 1H NMR spectrum which showed a singlet signal at $d(7.2-7.8) \mathrm{ppm}$.

All the newly synthesized Pyrimidine derivatives were evaluated for their biological activity by using Agar well diffusion method.

The antibacterial activity evaluated against gram-positive bacteria Staphylococcus aureus, by using agar well diffusion method. All the synthesized Pyrimidine derivatives and standard drugs were dissolved in DMSO and the concentrations of test compounds were adjusted to 25,50 , 100 , and $200 \mu \mathrm{g} / \mathrm{ml}$ and of standard drug Ampicillin. Pyrimidine derivatives inhibited the growth of bacterial 
strains produced a zone of inhibition from $10 \mathrm{~mm}$ to 18.89 $\mathrm{mm}$ for gram positive bacteria, Staphylococcus aureus along with $\mathrm{IC}_{50}$ values ranging from $61 \mu \mathrm{g} / \mathrm{ml}$.

\section{Conclusion}

The compounds containing six members of nitrogen are of high significance from biological and medical point of view. A variety of biological activities are there in the larger pyrimidine arrays. With different heterocycle scaffolds the fusion of pyrimidine moieties produces new compounds of hybrid heterocycles which have highly enhanced action. The various biological actions are included in it which includes anti-viral, anti-bacterial, anti-tubercular, anti microbial, anti protozoal, anti hypertensive, antihistaminic, pain killers or analgesics, and anti inflammatory etc. an important pharmacophore is there in the pyrimidine moiety which with the nucleic acid synthesis and function is seen to interact. In large amount of alkaloids, antimicrobial, antibiotics and drugs the nucleus of pyrimidine is present. Purines and pyrimidines are simply fused pyrimidines which by themselves are active from biological point of view and for some naturally occurring substrates they are very essential components such as nucleic acids. Pyrimethamine and Trimethoprim are some of the diamino pyrimidines which are strong anti malarial medicines and along with sulphonamides they are used in combination for better outcomes. This is also an antibacteriostatic which is potent. The pyrimidine moiety containing chemotherapeutic includes one of the most important suphadiazine. On the other hand, for the treatment of tuberculosis the nitrogen containing a lot of heterocycles are used such as Pyrazinamide and Clofazimine, Isoniazid etc. The structural precedence is offered by these compounds which along with analogues of pyrimidine as well as chalcone can end up in the generation of new therapeutics for tuberculosis. On the development of newer six member heterocyclic derivatives like pyrimidine and pyridine having antimycobacterial properties is the main focus of our research.

The new and improved artificial applicability as well as action of these heterocycles biologically are considered helpful for the pharmacists to plan, systematize and put into practice better and improved ways to find out and discover new series of drugs.

Among all the synthesized Pyrimidine derivatives ( $\mathrm{P} 1$ to P5), Pyrimidine derivatives P5 and shows good activity against Staphylococcus aureus and lesser toxicity.

\section{Conflict of Interest}

There doesn't includes any kind of conflict of interest while publishing this manuscript.

\section{References}

[1] David, A. Williams, and Thomas, L., 2002. Foye's principles of medicinal chemistry. Fifth edition, pp. 12-13.

[2] Remington, the science and practice of pharmacy Fifth edition, pp. 458.

[3] Copenhaver, A., and Kleinschmidt, B., 1952 Chemistry of heterocyclic compounds. British patent. Vol. 46. pp. 663, 303.

[4] Singh, H. and Kapoor, V. K., 2003. Medicinal and pharmaceutical chemistry, pp. 29.

[5] Agarwal, O. P., 2002 Organic chemistry, Reaction and Reagent, pp. 735.

[6] Jain, M. K., andSharnevas, S. C., 2006 Organic chemistry. Vol. 22, pp. 997-999.

[7] Sasada, T. Kobayashi, F. Sakai, N. Konakahara, T., 2009. Synthesis of pyrimidine. Organic Letters. Vol. 11, pp. 21612164.

[8] Ahmad, O. K. Hill, M. D. Movassaghi, M., 2009. Synthesis of pyrimidine. Journal of Organic Chemistry. Vol. 74, pp. 84608463.

[9] Barthakur, M. G. Borthakur, M. Devi, P. Saikia, C. JSaikia,. A. Bora, U. Chetia, A. Boruah, R. C., 2007. Synthesis of pyrazolopyrimidinederivativesSynthetic Organic Chemistry, 223-226.

[10] Movassaghi, M. Hill, M. D., 2006. Synthesis of pyrimidine derivatives. Journal of American Chemical Society. Vol. 128, pp. 14254-14255.

[11] Collins, A. H., 2009. Microbiological methods. London: Butterworth. Vol. 2, pp: 223-227.

[12] Carter, S. J., 1990. Cooper and Gunn's tutorial pharmacy Delhi: CBS publishers and distributors. Vol. 6, pp. 349.

[13] Jacob, V. Michael, A. 1999 Nutrition Vol. 49, pp. 1-17.

[14] Magaldi, S. Mata-Essayag, C. Capriles, H. D. Collela, M. T. Olaizola, C. 2004. Well diffusion for antifungal susceptibility testing. International Journal of Infectious Diseases. Vol. 8, pp. 39-45.

[15] Buege, J. A. Aust, S. D., 1978. Suppression of fatty acid synthase by dietary polyunsaturated fatty acids is mediated by fat itself, not by peroxidative mechanismJournal of Biochemistry and Molecular Biology. Vol. 30, pp. 302. 